\title{
Acute tubulointerstitial nephritis in 10-year-old boy following severe acute respiratory syndrome coronavirus 2 infection
}

\author{
Aleksandra Sobieszczańska-Droździel ${ }^{1}$, Adam Strzoda², Wojciech Sowiński ${ }^{2}$ \\ 'Department of Pediatric Nephrology, Medical University of Lublin, Poland \\ ${ }^{2}$ Student's Scientific Group at the Department of Pediatric Nephrology, Medical University of Lublin, Poland
}

\section{ABSTRACT}

The COVID-19 pandemic caused by coronavirus SARS-CoV-2 is currently a main public health problem worldwide. The clinical symptoms relate primarily to the respiratory system but may also involve multiple organs. The course of COVID-19 in children is usually mild, but in some cases may cause late complications, particularly the pediatric inflammatory multisystem syndrome (PIMS-TS). One of its symptoms may be acute kidney injury.

We present a 10-year-old boy who developed nonspecific symptoms a few weeks after mild COVID-19, including weakness, weight loss, and polyuria. Clinical evaluation revealed acute renal failure secondary to acute tubulointerstitial nephritis (ATIN). Treatment with glucocorticoids resulted in rapid clinical and laboratory improvement. We hypothesize that the development of ATIN could be causally related to COVID-19 in an immune pathomechanism similar to PIMS-TS. The case provides new insights into possible complications of SARS-CoV-2 infection and indicates the need for renal follow-up after COVID-19.

KEY WORDS:

COVID-19, acute tubulointerstitial nephritis, PIMS-TS.

\section{INTRODUCTION}

A pandemic of coronavirus disease 2019 (COVID-19) caused by the novel coronavirus SARS-CoV-2 (severe acute respiratory syndrome coronavirus 2), first identified in December 2019 in Wuhan, China, become a main public health problem worldwide. Although COVID-19 is primarily a pulmonary disease, it may involve all other organs and may lead to a fatal outcome. The clinical presentation in children is usually mild or asymptomatic [1-3], however serious post-COVID complications may occur in this age group [4].

We report on a 10 -year-old boy who after mild COVID-19 developed acute tubulointerstitial nephritis (ATIN) with renal failure. The clinical course may sug- gest a causative role of SARS-CoV-2 infection in this process.

\section{CASE REPORT}

A 10-year-old boy was referred at the end of December 2020 to our Department shortly after the renal failure of unknown origin was diagnosed at a local hospital. Ten weeks earlier the boy developed mild COVID-19 confirmed by real-time RT-PCR test, presented with shortterm fever, sore throat, myalgia, dry cough, and dysgeusia. He was not treated with antibiotics and only twice took a small dose of paracetamol. When within the next few weeks following recovery from acute symptoms, fatigue, decreased appetite, polyuria, and weight loss

\section{ADDRESS FOR CORRESPONDENCE:}

Aleksandra Sobieszczańska-Droździel, Department of Pediatric Nephrology, Medical University of Lublin,

Poland, e-mail: aleksandra.sobieszczanska-drozdziel@umlub.pl 
TABLE 1. Follow-up of selected laboratory results in the presented patient

\begin{tabular}{|c|c|c|c|c|c|c|}
\hline Laboratory parameters & $\begin{array}{l}\text { Reference } \\
\text { range }\end{array}$ & $\begin{array}{c}\text { Admission } \\
\text { (day 0) }\end{array}$ & $\begin{array}{l}\text { Prednisone } \\
\text { initiation } \\
\text { (day 9) }\end{array}$ & $\begin{array}{l}\text { Follow-up } \\
\text { (day 16, } \\
\text { prednisone } \\
30 \text { mg/day) }\end{array}$ & $\begin{array}{l}\text { Follow-up } \\
\text { (day 33, } \\
\text { prednisone } \\
30 \text { mg/day) }\end{array}$ & $\begin{array}{l}\text { Follow-up } \\
\text { (day 62, } \\
\text { prednisone } \\
10 \text { mg/day)) }\end{array}$ \\
\hline \multicolumn{7}{|l|}{ Blood } \\
\hline Urea, mg/dl & $15-36$ & 43.9 & 29.8 & 46.5 & 34.7 & 24.5 \\
\hline Creatinine, $\mathrm{mg} / \mathrm{dl}$ & $0.39-0.73$ & 1.31 & 1.26 & 0.78 & 0.64 & 0.64 \\
\hline Uric acid, mg/dl & $2.2-5.8$ & 3.37 & - & - & 2.79 & 3.59 \\
\hline C-reactive protein mg/dl & $0-0.5$ & 4.42 & 2.28 & 0.45 & 0.1 & 0.15 \\
\hline Procalcitonin, ng/ml & $0-0.5$ & 0.353 & - & - & 0.06 & 0.058 \\
\hline Ferritin ng/ml & $14-124$ & 320.8 & 279 & 245.7 & 103.8 & 70.1 \\
\hline Erythrocyte Sedimentation Rate, mm/h & $2-15$ & 131 & 120 & 118 & 17 & 16 \\
\hline Troponin l, ng/ml & $<0.16$ & $<0.1$ & $<0.1$ & $<0.1$ & $<0.1$ & $<0.1$ \\
\hline pro-BNP, pg/ml & $0-62$ & 209.1 & 884.6 & 143.1 & $<10$ & $<10$ \\
\hline Hematocrit, \% & $35-42.4$ & 29.7 & 29.7 & 31.5 & 35.1 & 40.9 \\
\hline Hemoglobin, g/dl & $12-14$ & 9.6 & 9.7 & 10.3 & 11.7 & 13.9 \\
\hline$M C V, f l$ & $76.5-90.6$ & 73.6 & 74.1 & 75 & 80.1 & 80 \\
\hline $\mathrm{MCH}, \mathrm{pg}$ & $25-31$ & 25.3 & 24.2 & 24.5 & 26.7 & 27.2 \\
\hline Platelet count, $\mathrm{n} \times 10^{3}$ cells $/ \mu \mathrm{l}$ & $140-420$ & 766 & 679 & 911 & 338 & 445 \\
\hline Albumin, g/dl & $3.8-5.4$ & 3.9 & 4.23 & 4.59 & 5.06 & 5.04 \\
\hline Total protein, g/dl & $6-8$ & 8,4 & 9,05 & 8.82 & 7,97 & 7.79 \\
\hline Alanine aminotransferase, $\mathrm{U} / \mathrm{I}$ & $0-39$ & 8 & - & - & 26 & 22 \\
\hline Immunoglobulin G, mg/dl & $698-1560$ & 2013 & 2053 & - & 1090 & 1057 \\
\hline Immunoglobulin A, mg/dl & $53-204$ & 419 & 386 & - & 172 & 153 \\
\hline Complement C3, mg/dl & $80-150$ & 147 & 158 & - & 124 & 138 \\
\hline Complement C4, mg/dl & $12-36$ & 35 & 39 & - & 22 & 27 \\
\hline Fibrinogen, g/l & $1.9-4.0$ & 6.04 & 5.73 & 4.12 & 2.41 & 3.55 \\
\hline D-dimers, ng/ml & $<500$ & 1084 & 782 & 431 & 366 & 354 \\
\hline Calcium, mmol/l & $2.2-2.7$ & 2.54 & 2.59 & 2.63 & 2.58 & 2.59 \\
\hline Phosphates, mmol/l & $1.05-1.85$ & 1.82 & 1.48 & 1.4 & 1.31 & 1.56 \\
\hline Bicarbonates, mmol/l & $22.5-30$ & 20.5 & 21 & 21.6 & 22.4 & 22 \\
\hline \multicolumn{7}{|l|}{ Urine } \\
\hline Specific gravity & $1.015-1.030$ & 1.010 & 1.005 & 1.010 & 1.025 & 1.015 \\
\hline Glucose, mg/dl & 0 & 0 & 85 & 168 & 0 & 0 \\
\hline Protein, mg/dl & $0-15$ & 0 & 0 & 0 & 0 & 0 \\
\hline $\mathrm{pH}$ & $5-7$ & 5.0 & 6.5 & 8.0 & 6.0 & 7.0 \\
\hline Erythrocytes $n$ per high power field & $0-5$ & $0-1$ & 0 & 0 & 0 & 0 \\
\hline Leukocytes, n per high power field & $0-5$ & $3-5$ & $0-1$ & $1-3$ & $2-4$ & $0-1$ \\
\hline Uric acid excretion, mg/kg/24 h & $5-15$ & - & - & - & 22.8 & 18.63 \\
\hline Protein excretion, mg/24 h & $0-140$ & - & - & - & $<30$ & $<30$ \\
\hline
\end{tabular}

of about eight kilograms were observed, he was admitted to the local hospital. Laboratory tests showed positive anti-SARS CoV2 antibodies titer (179.4 COI, negative $<1.0$ ), negative SARS-CoV-2 nucleoprotein based antibody test, elevated serum creatinine level $(1.4 \mathrm{mg} / \mathrm{dl}$,
eGFR $58.3 \mathrm{ml} / \mathrm{min} / 1.73 \mathrm{~m}^{2}$, estimated with Schwartz formula) with normal urea $(42 \mathrm{mg} / \mathrm{dl})$ and relatively low uric acid (3.2 mg/dl) concentrations, increased inflammatory markers (CRP $4.42 \mathrm{mg} / \mathrm{dl}$, ESR $131 \mathrm{~mm} / \mathrm{h}$, ferritin $307.71 \mathrm{ug} / \mathrm{l}$ ), moderate anemia (Hgb $10.3 \mathrm{~g} / \mathrm{dl}$, 
Hct $31.1 \%)$, thrombocytosis $\left(750 \times 10^{3} / \mathrm{ul}\right)$ and reduced serum bicarbonates $(19.3 \mathrm{mmol} / \mathrm{l})$. Urinalysis was normal. Chest X-ray was without pathological findings but abdominal ultrasound (USG) revealed bilaterally enlarged kidneys with parenchymal thickening.

On admission to our Department, the patient was in good condition but with significant weakness and fatigue, normal body temperature, and blood pressure of 121/81 mm Hg. Physical examination showed only skin pallor, slightly positive, bilateral Goldflam sign, and mild tenderness in the upper abdomen. The results of laboratory tests confirmed decreased renal function with eGFR $59.62 \mathrm{ml} / \mathrm{min} / 1.73 \mathrm{~m}^{2}$, increased levels of inflammatory parameters, normal urinalysis, and moderate anemia. Besides, hypergammaglobulinemia, moderately elevated serum pro-BNP and D-dimer were revealed. Serum levels of C3 and C4 of complement, $\mathrm{LDH}$, cardiac, and liver enzymes were normal (Table 1). USG showed enlarged, hyperechoic kidneys with loss of corticomedullary differentiation (Figure 1). Urine culture, serological tests for cytomegalovirus (CMV), hepatitis $B$ virus (HBV), hepatitis $\mathrm{C}$ virus (HCV), Epstein-Barr virus (EBV), toxoplasmosis, and borreliosis, as well as antinuclear antibodies, c-ANCA, and p-ANCA were negative. The patient presented with polyuria due to failed urine concentration ability (hyposthenuria) with daily diuresis between 3,5 and 5 liters. Six days later, he developed glycosuria. As ATIN, as a reason for acute kidney injury (AKI) was suspected, symptomatic treatment with parenteral fluid infusion and oral sodium bicarbonate supplementation was started. Due to elevated serum D-dimer level, low-molecular-weight heparin as thromboprophylaxis was injected in a single daily dose. In follow-up, serum level of pro-BNP increased to maximally $884.6 \mathrm{pg} / \mathrm{ml}$, but troponin I, echocardiography, and electrocardiography remained normal. As in the following 8 days, no significant clinical or laboratory improvement was observed, treatment with oral prednisone in a daily dose of $1 \mathrm{mg} / \mathrm{kg}$ was introduced for 4 weeks. As a result, a quick improvement, with normalization of kidney function (eGFR $100 \mathrm{ml} / \mathrm{min} / 1.73 \mathrm{~m}^{2}$ ) and CRP level was observed (Table 1, Figure 2) and the patient was discharged home.

A follow-up, performed 2 weeks later, showed resolution of fatigue, an increase of appetite, weight gain of more than three kilograms, normal eGFR, resolution of glycosuria, and normalization of renal imaging (Table 1, Figure 2). Thereafter, the prednisone dose was stepwise reduced by $5 \mathrm{mg}$ every week without any negative impact on the patient's results (Table 1, Figure 2).

\section{DISCUSSION}

Acute tubulointerstitial nephritis is a rare cause of AKI in children, accounting for less than $10 \%$ of all cases and involving the renal tubulointerstitial tissue with an infiltrate of T-lymphocytes, eosinophils, and monocytes, while glomeruli and vessels are initially unaffected. Since in the majority of cases renal biopsy is not performed, the di-

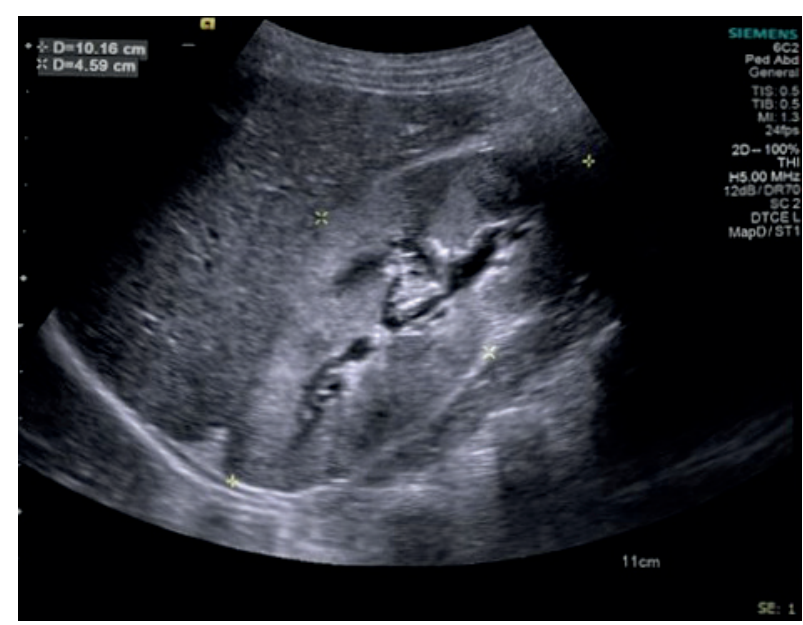

FIGURE 1. Renal ultrasound of the right kidney in the presented patient. The kidney is enlarged $(101.6 \times 45,9 \mathrm{~mm})$, hyperechoic with partial loss of corticomedullary differentiation

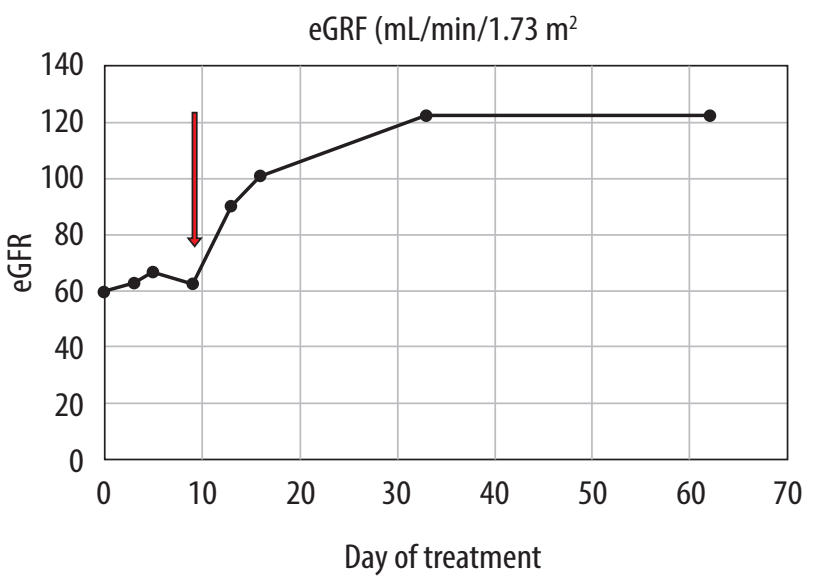

FIGURE 2. Follow-up of eGFR in the presented patient (arrow indicates the first day of prednisone treatment)

agnosis of ATIN as in our patient is made on a clinical basis. Typical features include non-oliguric acute renal failure of various degrees, signs of tubular dysfunction, proteinuria and/or sterile leukocyturia, eosinophilia, increased renal echogenicity in USG, abdominal and/or loin pain, and normal blood pressure $[5,6]$. Our patient met most of these criteria, including non-oliguric AKI, renal glycosuria, a tendency to hypouricemia due to hyperuricosuria, decreased serum bicarbonate level, hyposthenuria, clinical symptoms, and typical renal imaging. Due to the patient's stable general condition, lack of progression of renal failure, and a very good response to steroid treatment we abandoned the initially planned kidney biopsy.

Acute tubulointerstitial nephritis is usually caused by hypersensitivity reactions to medications, toxins, autoimmune disorders, or infections. As these three former reasons may be rather excluded in our patient by his medical history and laboratory results, the only possible detectable factor remained SARS-CoV-2 infection.

It is well known that different microorganisms, including viruses and bacteria, may be responsible for the devel- 
opment of ATIN. Viral and bacterial antigens may initiate cell-mediated injury due to delayed idiosyncratic hypersensitivity immune reactions. The antigens are filtered, concentrated, and secreted within the kidneys what along with high blood flow, increases their exposure and makes them a target of immunological reactions [5, 6]. Respiratory viruses can trigger Kawasaki disease [7] while bacterial superantigens of either Staphylococcus aureus or Streptococcus pyogenes may cause toxic shock syndrome [8].

Acute kidney injury is the most common renal manifestation in COVID-19 patients, reported in around 3-9\% of them [9], but its incidence is much higher among those who require hospitalization [10]. The pathomechanism is not fully understood but it may be related to direct renal tropism of SARS-CoV-2 or indirect effects resulting from systemic consequences of COVID-19 [11]. The former is possible, as SARS-CoV-2 cellular membrane receptors - ACE2 are expressed in renal proximal tubular cells, podocytes, and glomerular visceral epithelial cells. It was confirmed by the presence of SARS-CoV-2 RNA in the damaged tubular epithelium [9]. Indirect mechanisms of AKI include renal hypoperfusion due to hypovolemia, cytokine storm, microvascular thrombosis, or side effects of intensive treatment [12]. Interestingly, to date, only one case of biopsy-proven ATIN with COVID-19 and AKI was reported [13].

Specifically, in children AKI related to COVID-19 may be a part of pediatric inflammatory multisystem syndrome temporally associated with SARS-CoV-2 (PIMS-TS) [4, 14]. This serious condition usually occurs 2-6 weeks after COVID-19 as severe hyperinflammation leading to multisystemic manifestation. It is characterized by a broad spectrum of symptoms, including fever, myalgia, myocarditis, coronary aneurysms, gastroenteritis, lymphadenopathy, skin rash, stomatitis, conjunctivitis, central neuropathy, pneumonia, elevated markers of inflammation, and renal failure. Although precise pathomechanism of PIMS-TS is not fully understood, it is considered as a post-viral abnormal immunological reaction $[4,15]$.

Obviously, the presented patient did not meet the diagnostic criteria of PIMS-TS. However, we cannot exclude the immunological mechanism of ATIN in this case. Similarly to PIMS-TS, our patient developed a systemic inflammatory reaction confirmed by elevated CRP, ESR, ferritin, fibrinogen, D-dimer levels, as well as hypergammaglobulinemia. It may be responsible for his general symptoms, including malaise and severe weight loss several weeks before clinical evaluation. If kidneys and other organs were involved in this process during this time, remains speculative. Finally, the rapid response to corticosteroids may also support our hypothesis.

\section{CONCLUSIONS}

The presented case shows a possible complication of SARS-CoV-2 infection and provides new insights into the clinical spectrum of COVID-19. We hypothesize that the development of ATIN after COVID-19 may represent a milder form of PIMS-TS, which in some cases may be overlooked. Therefore, a follow-up of pediatric cases with prior COVID-19, regardless of its severity, should be warranted.

\section{DISCLOSURE}

The authors declare no conflict of interest.

\section{REFERENCES}

1. Mania A, Mazur-Melewska K, Lubarski K, et al. Wide spectrum of clinical picture of COVID-19 in children - from mild to severe disease. J Infect Public Health 2021; 14: 374-379.

2. Jackowska T, Peregud-Pogorzelski J, Marczyńska M, et al. Recommendations of the Polish Paediatric Society and the National Consultant in the field of paediatrics regarding outpatient care for children during the COVID-19 pandemic caused by SARS-CoV-2. Pediatr Pol 2020; 95: 61-64.

3. Jackowska T, Marczyńska M, Peregud-Pogorzelski J. Guidance for paediatric emergency departments/rooms and departments of paediatrics on the management of a child suspected of or diagnosed with COVID-19. Pediatr Pol 2020; 95: 65-67.

4. Harwood R, Allin B, Jones CE, et al. A national consensus management pathway for paediatric inflammatory multisystem syndrome temporally associated with COVID-19 (PIMS-TS): results of a national Delphi process. Lancet Child Adolesc Health 2021; 5: 133-141.

5. Roy S, Awogbemi T, Holt RCL. Acute tubulointerstitial nephritis in children - a retrospective case series in a UK tertiary paediatric centre. BMC Nephrol 2020; 21: 17.

6. Joyce E, Glasner P, Ranganathan S, et al. Tubulointerstitial nephritis: diagnosis, treatment, and monitoring. Pediatr Nephrol 2017; 32: 577-587.

7. Turnier JL, Anderson MS, Heizer HR, et al. Concurrent respiratory viruses and Kawasaki disease. Pediatrics 2015; 136: e609-614.

8. McCormick JK, Yarwood JM, Schlievert PM. Toxic shock syndrome and bacterial superantigens: an update. Ann Rev Microbiol 2001; 55: 77-104.

9. Mubarak M, Nasri H. COVID-19 nephropathy; an emerging condition caused by novel coronavirus infection. J Nephropathol 2020; 9: e21.

10. Fabrizi F, Alfieri CM, Cerutti R, et al. COVID-19 and Acute Kidney Injury: A Systematic Review and Meta-Analysis. Pathogens 2020; 9: 1052 .

11. Nadim MK, Forni LG, Mehta RL, et al. COVID-19-associated acute kidney injury: consensus report of the 25th Acute Disease Quality Initiative (ADQI) Workgroup. Nat Rev Nephrol 2020; 16: 747-764.

12. Meijers B, Hilbrands LB. The clinical characteristics of coronavirus-associated nephropathy. Nephrol Dial Transplant 2020; 35: 1279-1281.

13. Szajek, K, Kajdi ME, Luyckx VA, et al. Granulomatous interstitial nephritis in a patient with SARS-CoV-2 infection. BMC Nephrol 2021; 22: 19.

14. Lawrensia S, Henrina J, Wijaya E, et al. Pediatric Inflammatory Multisystem Syndrome Temporally Associated with SARS-CoV-2: a New Challenge amid the Pandemic. SN Compr Clin Med 2020; 2: 2077-2085

15. Esposito S, Principi N. Multisystem Inflammatory Syndrome in Children Related to SARS-CoV-2. Pediatr Drugs 2021; 23: 119-129. 